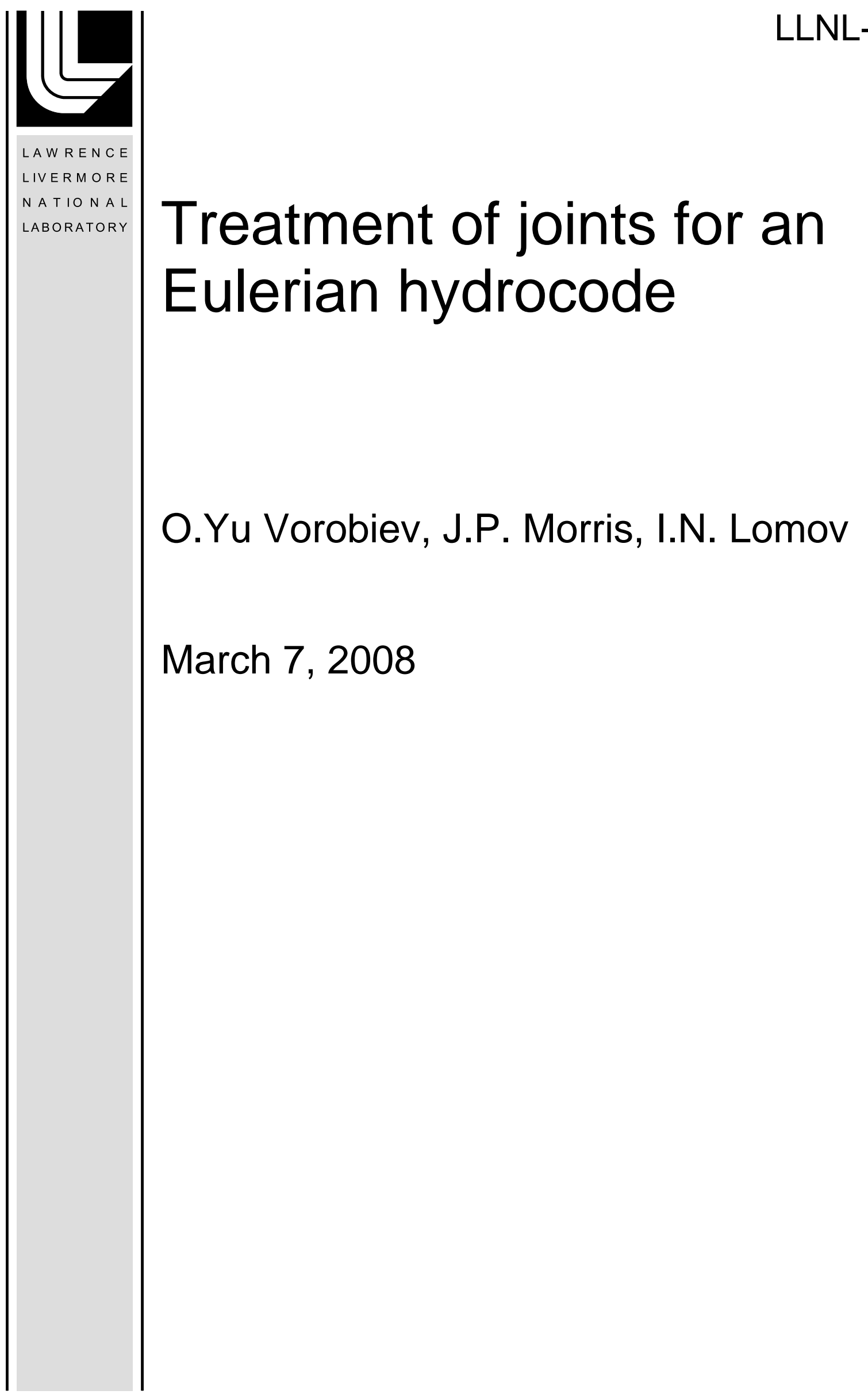


This document was prepared as an account of work sponsored by an agency of the United States government. Neither the United States government nor Lawrence Livermore National Security, LLC, nor any of their employees makes any warranty, expressed or implied, or assumes any legal liability or responsibility for the accuracy, completeness, or usefulness of any information, apparatus, product, or process disclosed, or represents that its use would not infringe privately owned rights. Reference herein to any specific commercial product, process, or service by trade name, trademark, manufacturer, or otherwise does not necessarily constitute or imply its endorsement, recommendation, or favoring by the United States government or Lawrence Livermore National Security, LLC. The views and opinions of authors expressed herein do not necessarily state or reflect those of the United States government or Lawrence Livermore National Security, LLC, and shall not be used for advertising or product endorsement purposes.

This work performed under the auspices of the U.S. Department of Energy by Lawrence Livermore National Laboratory under Contract DE-AC52-07NA27344. 


\title{
Treatment of joints for an Eulerian hydrocode
}

\author{
Oleg Vorobiev, Ilya Lomov and Joseph Morris
}

\section{Introduction}

The treatment of discontinuities such as joints and faults is a key ingredient in any code used to model rock masses. The following study was done to improve treatment of discontinuities (joints) in the Eulerian hydrocode GEODYN [1-2]. In general, such discontinuities will introduce an increase in normal compliance in addition to a reduction in shear strength. In the present work we consider the limiting case of stiff discontinuities that only affect the shear strength of the material. In order to model such joints, a thin layer of the joint material with the same compressibility as the material around is added to the mesh cells where the joints are located. Thus, the joints have finite thickness which may change in time due to numerical diffusion. The joints are initialized using their volume fractions described by the corresponding internal variables. These variables are advected at every time step.

To resolve the joint the cells size should be smaller than the joint thickness. The strength of the material in the cells containing the joint material is limited by a value proportional to the pressure with a coefficient of proportionality corresponding to the coefficient of friction. An additional plastic update was used to limit the deviatoric stresses in those cells.

To investigate the accuracy of this joint treatment the GEODYN calculations have been compared with results from the Lagrangian code GEODYN-L which uses an explicit treatment of joints via common plane contact [3].

\section{Joint treatment in GEODYN}

Since the joint size typically is much smaller then the characteristic problem size, it is not always possible to allocate enough numerical cells to resolve the joint (see Fig. 1 case I). Yet, in advanced codes, like GEODYN, it is possible to use AMR techniques to resolve the most important joints (see Fig1. case II). The goal of the present study is to establish how many cells is needed to model the main effects of joints and how simple the model for the joints can be to reproduce those effects.

In the current version of GEODYN each joint, $i$, is characterized by its volume fraction, $f_{i}$, material $i d$ and the internal angle of friction, $\phi_{i}$. If the minimum and the maximum principal stresses are known, then

$$
\mu=\sin (\phi)=\frac{\sigma_{1}-\sigma_{3}}{\left(\sigma_{1}+\sigma_{3}\right)}
$$

In the case of triaxial loading the yield surface can be expressed as a linear function of pressure as

$$
\begin{aligned}
& Y=\alpha P \\
& \alpha=\frac{\partial Y}{\partial P}=\frac{6 \mu}{3-\mu}
\end{aligned}
$$


If many joints intersect the cell (as it is shown in Fig1. case I for cell A), the effective slope of the yield surface, $\alpha_{e f f}$ is found as

$$
\alpha_{e f f}=\frac{\sum f_{i}}{\sum \frac{f_{i}}{\alpha_{i}}},
$$

The joint material is treated the same way as the material with corresponding $i d$ except for one additional step: After the new stress is found, it is checked for consistency with the yield surface for the joints. So, the new von Mises stress, $\sigma_{e}$ should satisfy

$$
\sigma_{e}<\alpha_{e f f} p,
$$

where $p$ is the average pressure in the cell. The radial return algorithm is used to reduce the deviatoric stress if this condition is violated.

\section{Joint treatment in GEODYN-L}

GEODYN-L is a Lagrangian code where the discontinuities can be explicitly included as distinct contact elements. The following function of form is used for the normal modulus on the contact element:

$$
E=\left\{\begin{array}{c}
E_{0} \frac{a^{2}}{(a-u)^{2}}, \dot{u}>0 \\
E_{0} \frac{a^{2}}{\left(a-u_{\max }\right)^{2}}, \dot{u} \leq 0
\end{array}\right.
$$

Here $a$ is the aperture, $u$ is the normal closure, $E_{0}$ is the initial normal modulus and $u_{\max }$ is the maximum closure up to the current time. If integrated for the loading condition it gives the following dependence for the normal stress

$$
\sigma_{n}=\frac{E_{0} u}{(a-u)}
$$

Contacts are considered to be isotropic with a Coulomb friction law and a limited tensile strength. Thus the shear stress at the contact is limited by the yield surface dependent on the normal stress as

$$
\sigma_{s \max }=C+\sigma_{n} \tan (\phi)
$$

where $\mathrm{C}$ is the shear cohesion and $\phi$ is the friction angle. For stiff, noncompliant joints the aperture will be small compared to the cell size.

More detailed description of GEODYN-L is gived in [3]. 
case I
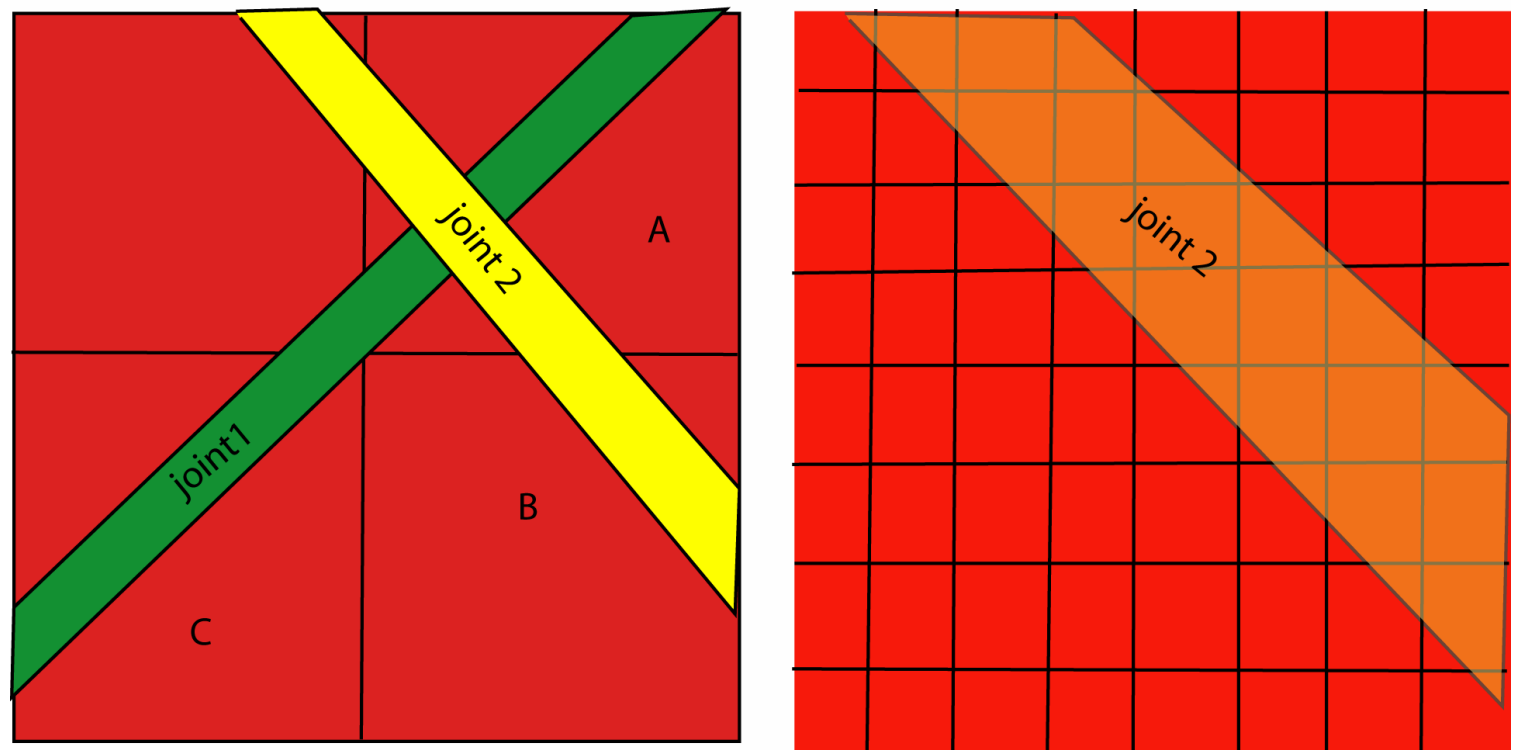

Figure .1 Possible cases for joints treatment: case I - under-resolved joints, case II resolved joint.

\section{TEST \#1: Impact of a square block with a diagonal joint onto a rigid wall}

This problem represents a case of joint orientation where the joint is not aligned with the Cartesian mesh. At the same time this problem is simple enough for the verification. The impact velocity was $100 \mathrm{~m} / \mathrm{s}$ along the $\mathrm{Y}$ axis. The rigid wall boundary was set at the top of the mesh (see Fig.2). The symmetry boundaries are set from the sides. 


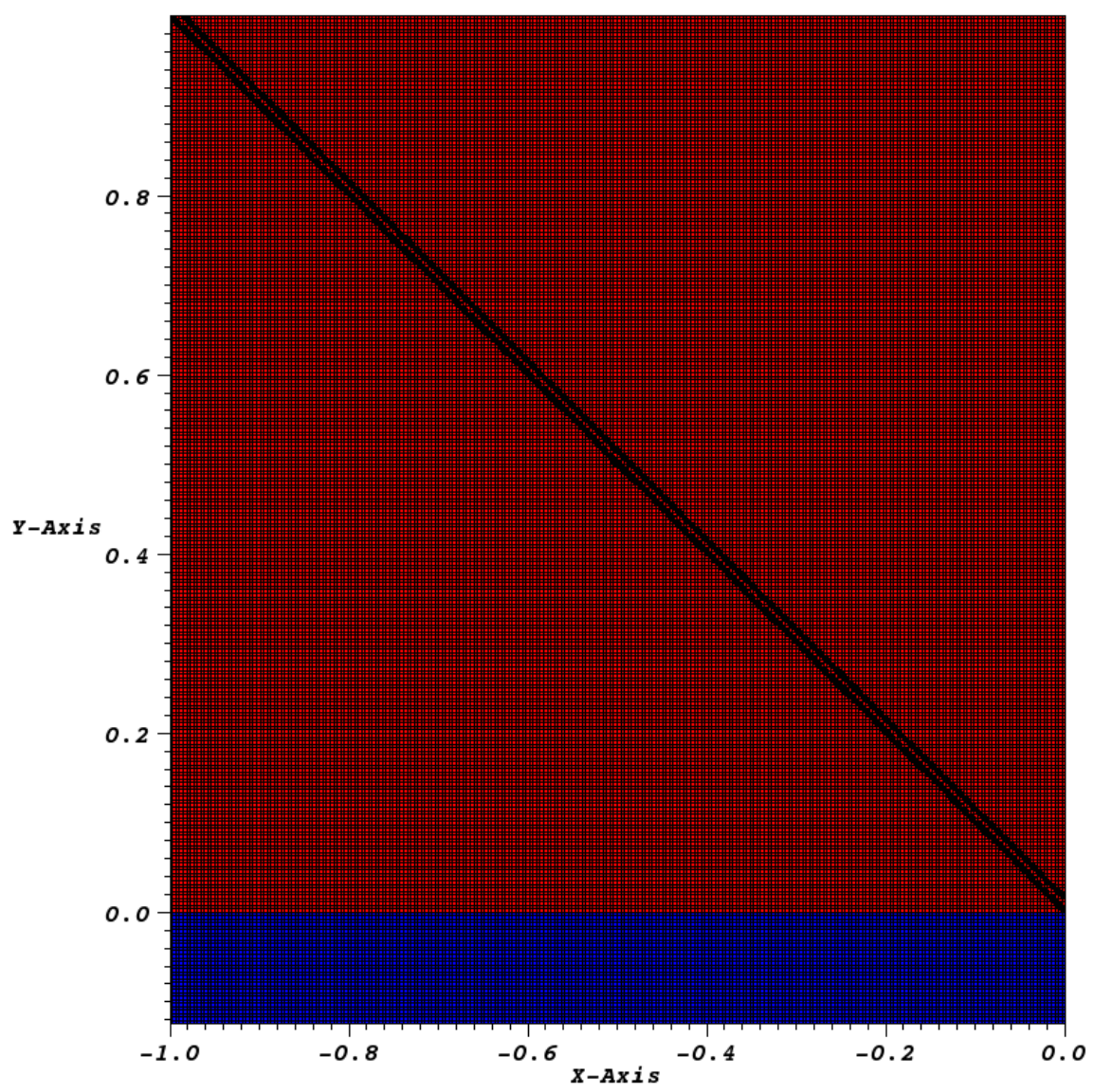

Figure. 2 Problem set-up with Geodyn (128x144 cells filled with 3 materials: air, concrete, and the joint material)

A more accurate representation of the joint is provided by the Lagrangian code where the joint was aligned with the mesh boundary (see Fig.3). The joint aperture, $a$, was $0.001 \mathrm{~m}$ to approximate a very stiff (practically incompressible) joint. 


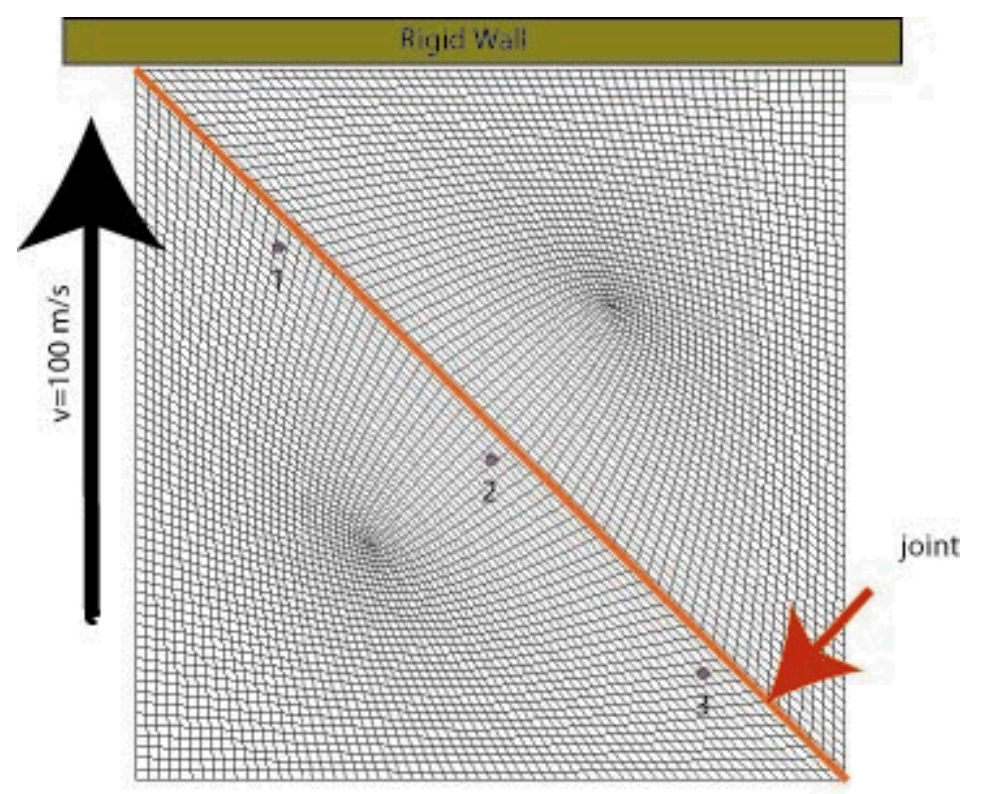

Figure .3 Problem set-up with Geodyn-L (two triangular regions meshed into 3072 quads)

It was found that the response of the joint in the Eulerian problem was mesh sensitive if the joint is under-resolved. Figure 4 shows the calculated horizontal velocities at the target points 1,2 , and 3 for two joint thicknesses $0.01 \mathrm{~m}$ (green lines) and $0.02 \mathrm{~m}$ (red lines). The frictional coefficient used in these calculations was $\phi=10$. The thinner the joint the more cells are needed for the converged solution. There should be 4-5 cells across the joint thickness in order to get the converged solution. Under-resolved joints tend to be more frictional than the resolved ones.

Figures 5-6 show comparison between the two codes for frictionless joints. It is clear that resolution is good enough such joints (noncompliant and frictionless) present the easiest case for GEODYN. Since the codes use different models for the joints, the results may differ even if the same internal friction angle is used in both codes. The best agreement can be obtained for quasi hydrostatic loading where the difference between the pressure and the normal stress at the joint is minimal. 
Friction angle $=10$

joint width $=0.01 \mathrm{~m}$

joint width $=0.02 \mathrm{~m}$

increased resolution:

(256 $\times 288$ for $0.02 \mathrm{~m}$ joint and $384 \times 432$ for $0.01 \mathrm{~m}$ joint)
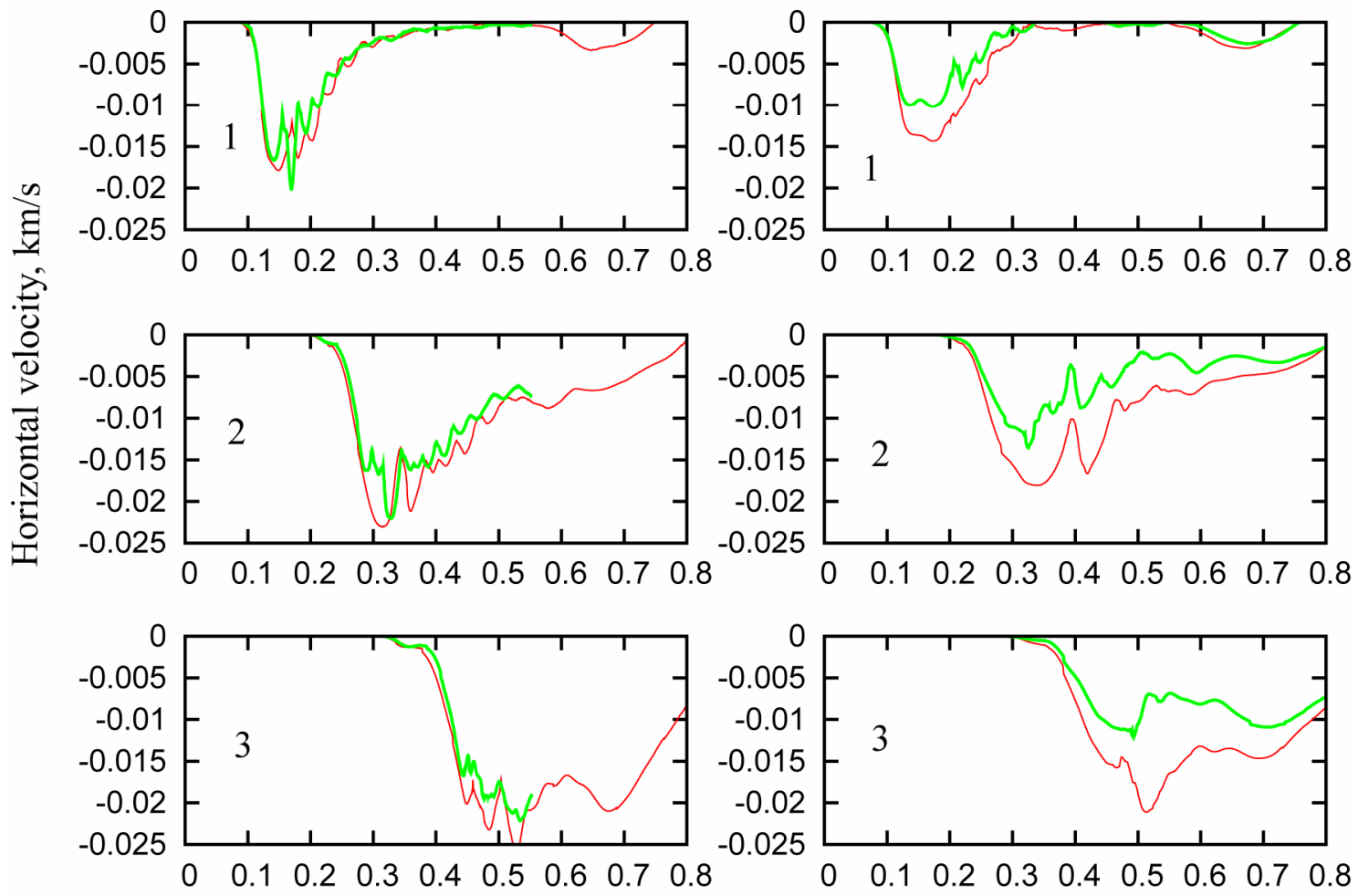

Time, $m s$

Figure.4 Convergence study with Geodyn joints for a friction angle of $\mathbf{1 0}$ 


\section{no friction}

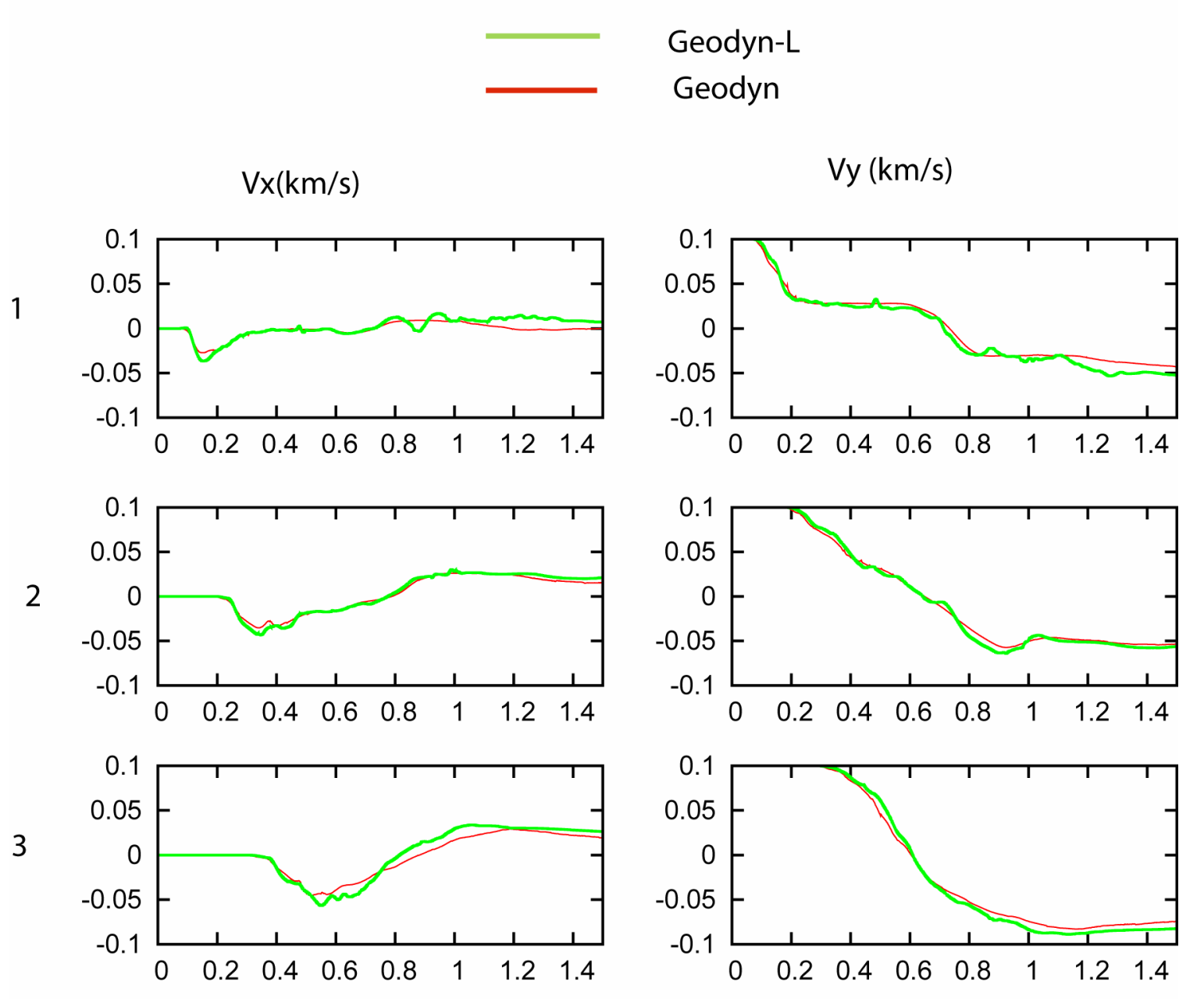

Time, $\mathrm{ms}$

Figure.5 Comparison of Geodyn-L and Geodyn for frictionless joint 


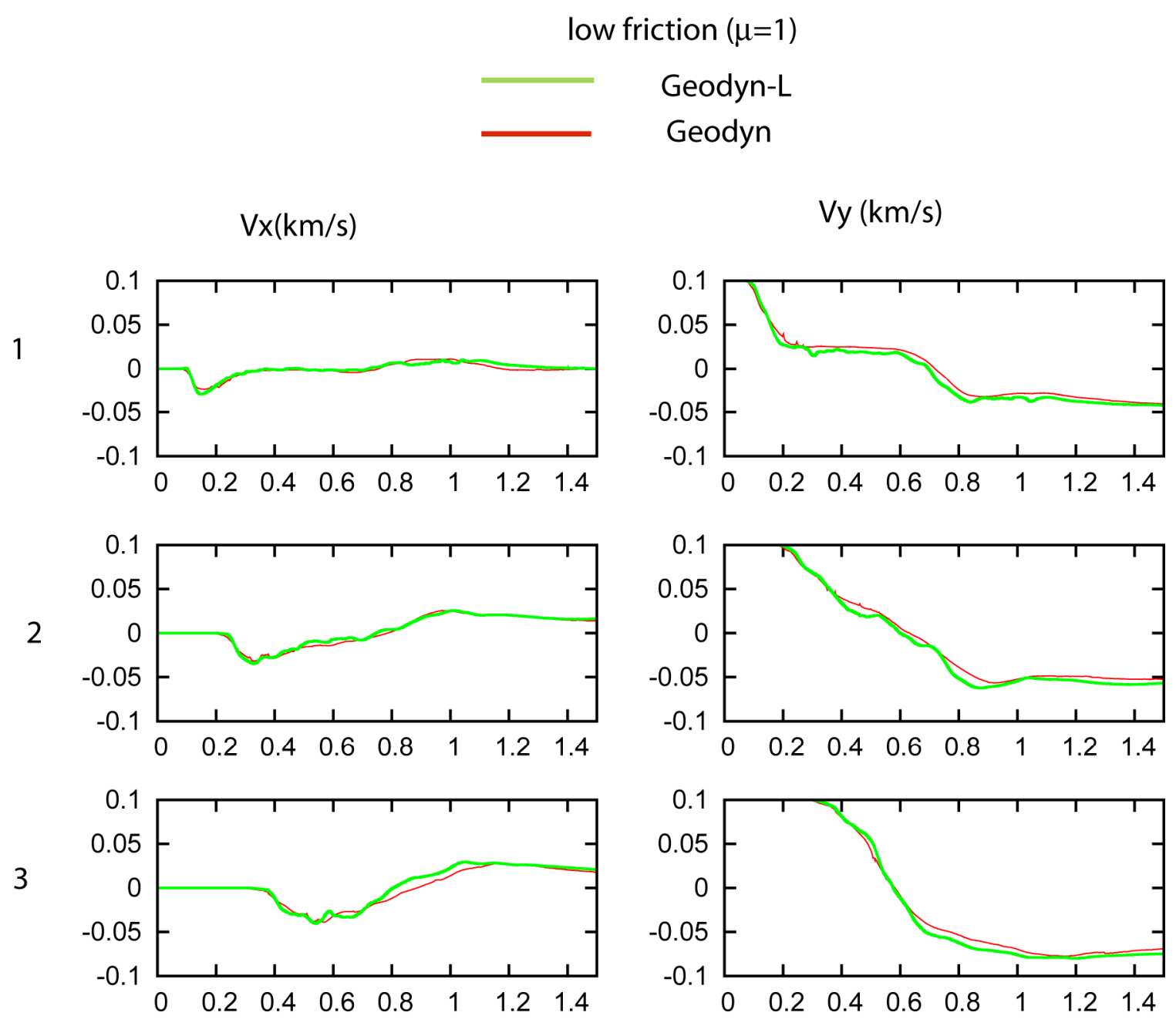

Time, ms

Figure.6 Comparison of Geodyn-L and Geodyn for a friction angle of 1 


\section{TEST \#2: Impact of a triangular block with multiple diagonal joints onto a rigid wall}

The main purpose of this problem is to test how GEODYN treats multiple joints which are not aligned with the Eulerian grid. The problem set-up is similar to TEST\#1 but 9 oblique joints were used instead of one. The material was initialized with a velocity of $100 \mathrm{~m} / \mathrm{s}$. The region was a $1 \mathrm{~m}$ by $1 \mathrm{~m}$ cube with one half filled with concrete and the other half filled with air. Four target points were used to monitor the flow parameters (velocities and stresses). Three target points $(0,1,2)$ were located at the material boundary, and one point (3) was located inside the jointed region. As the shock wave running from the wall reaches the concrete-air interface it turns into an unloading wave which releases the load from the joints. Consequently, the joint model was tested for both loading and unloading.

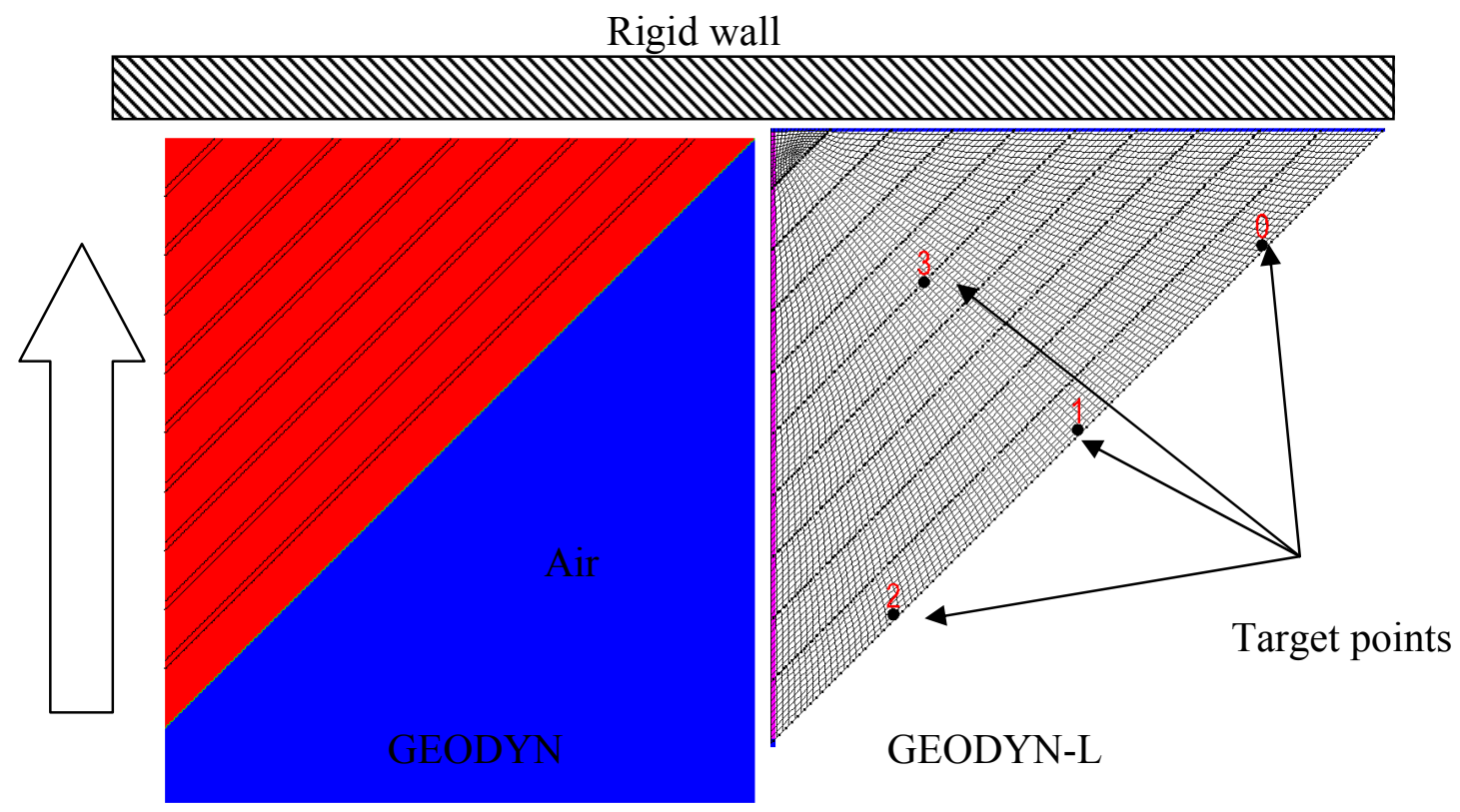

Figure.7 Problem set-up for GEODYN and GEODYN-L

Figures 8-10 show the comparison between the GEODYN and GEODYN-L calculations for the vertical velocity at target points (0-4). The friction angle was changed from 10 to 60 degrees. The model used in GEODYN assumes a triaxial stress state, therefore, results may differ for more general cases. Figure 11 shows that the agreement between the codes can be improved if a modified friction angle is used in GEODYN. 


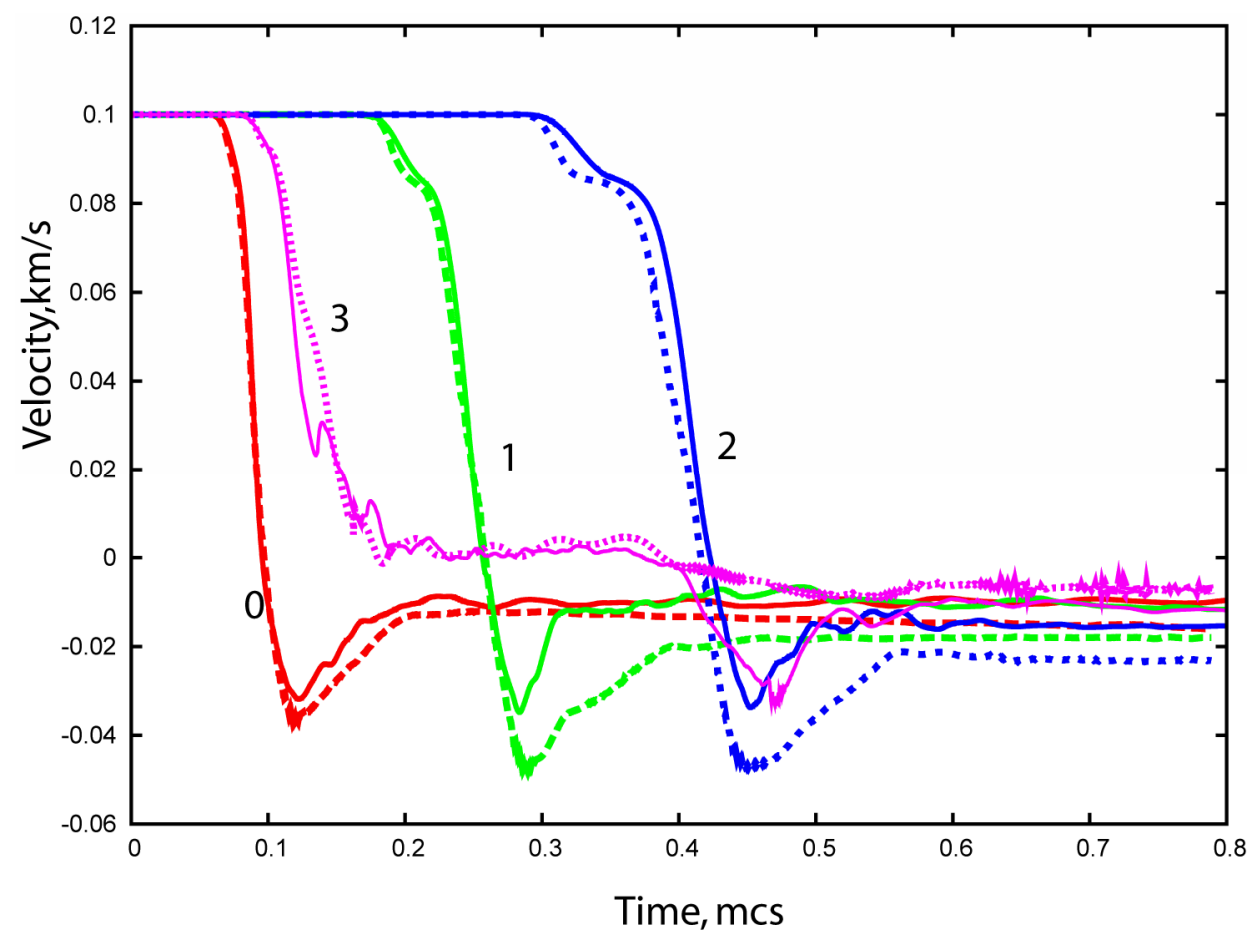

Figure. 8 Calculated vertical velocity histories. The friction angle is 30 . The solid lines are Geodyn-L calculations, the dashed lines are GEODYN calculations.

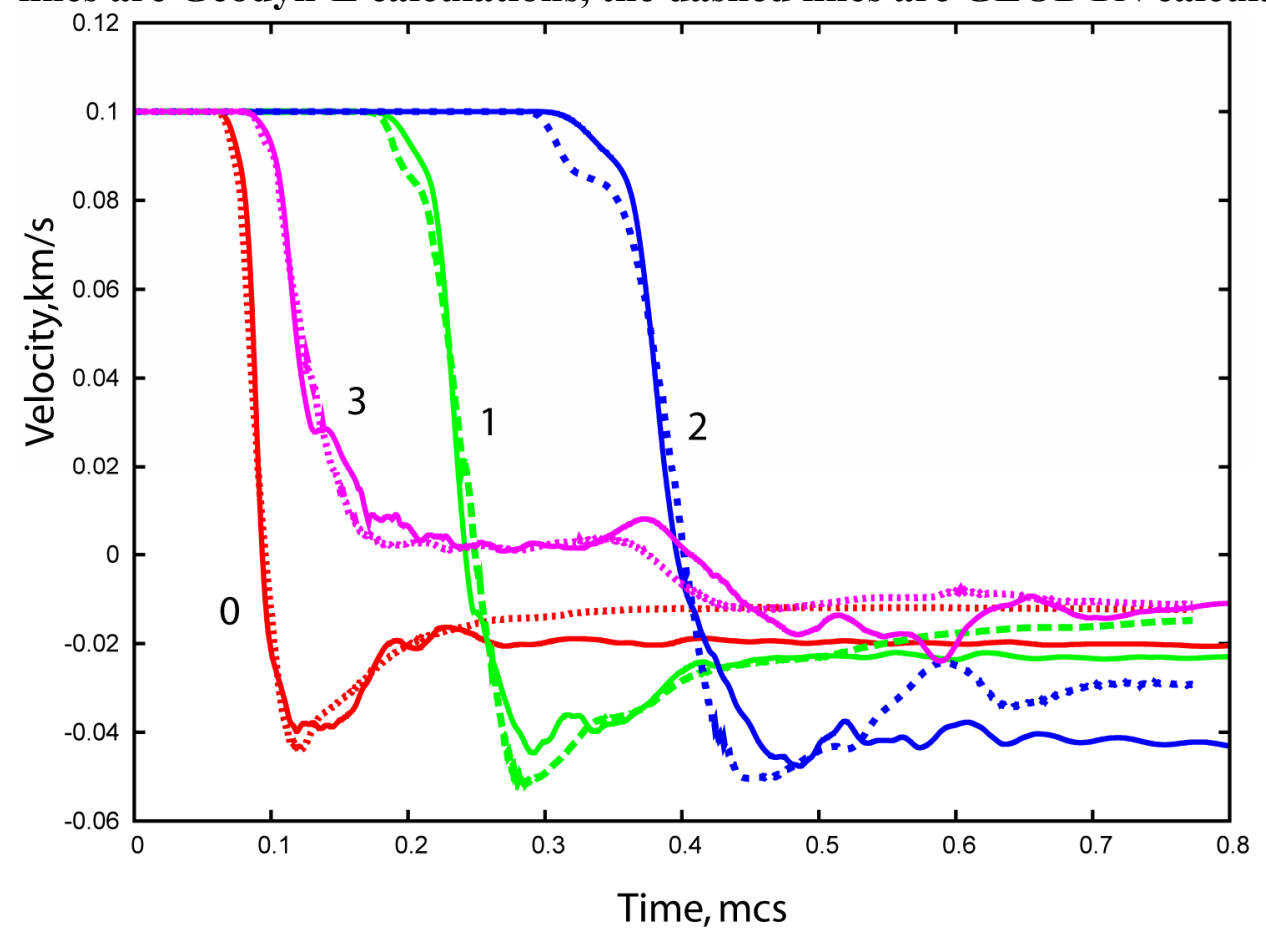

Figure.9 Calculated vertical velocity histories. The friction angle is 60 . The solid lines are Geodyn-L calculations, the dashed lines are GEODYN calculations. 


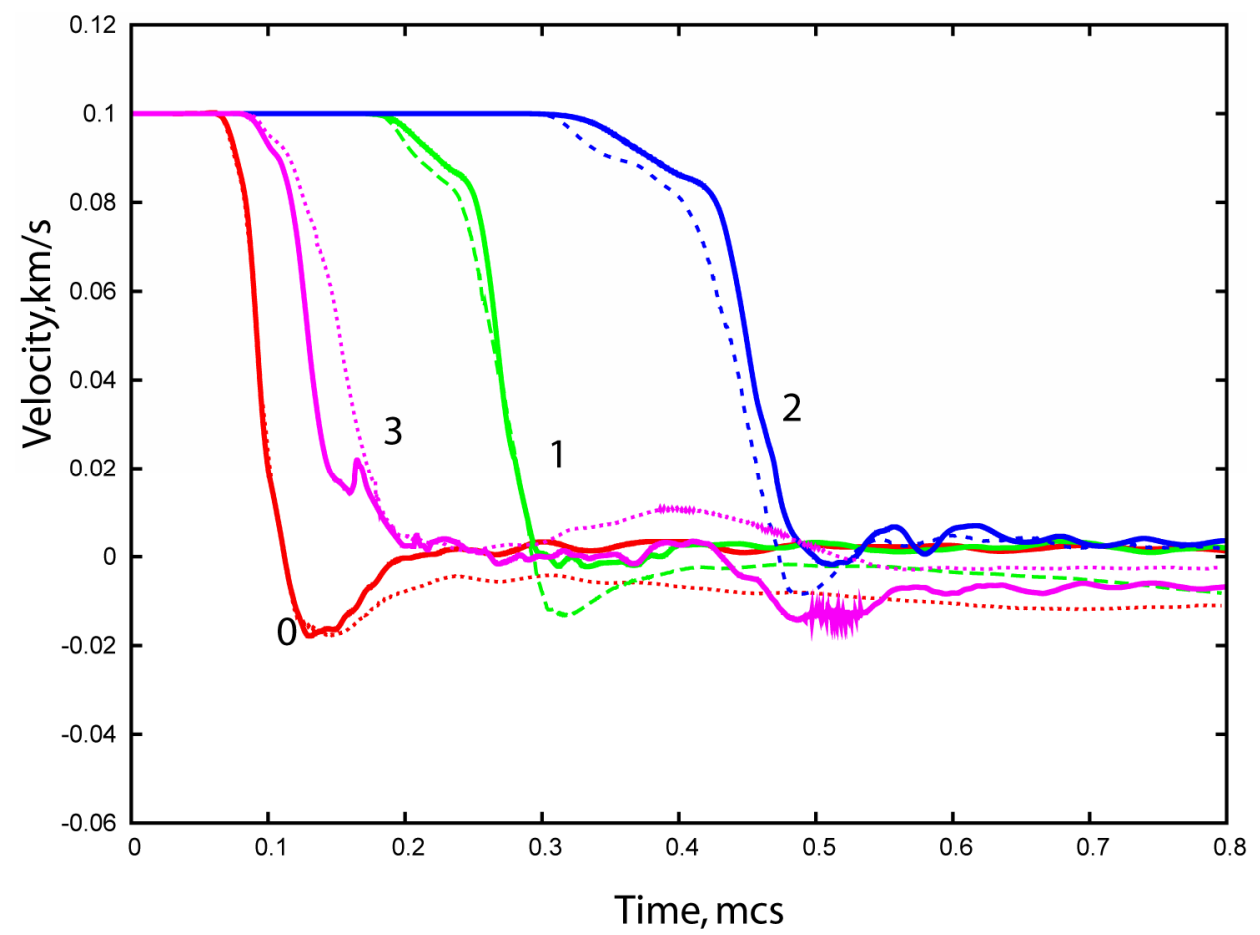

Figure.10 Calculated vertical velocity histories. The friction angle is 10 . The solid lines are Geodyn-L calculations, the dashed lines are GEODYN calculations.

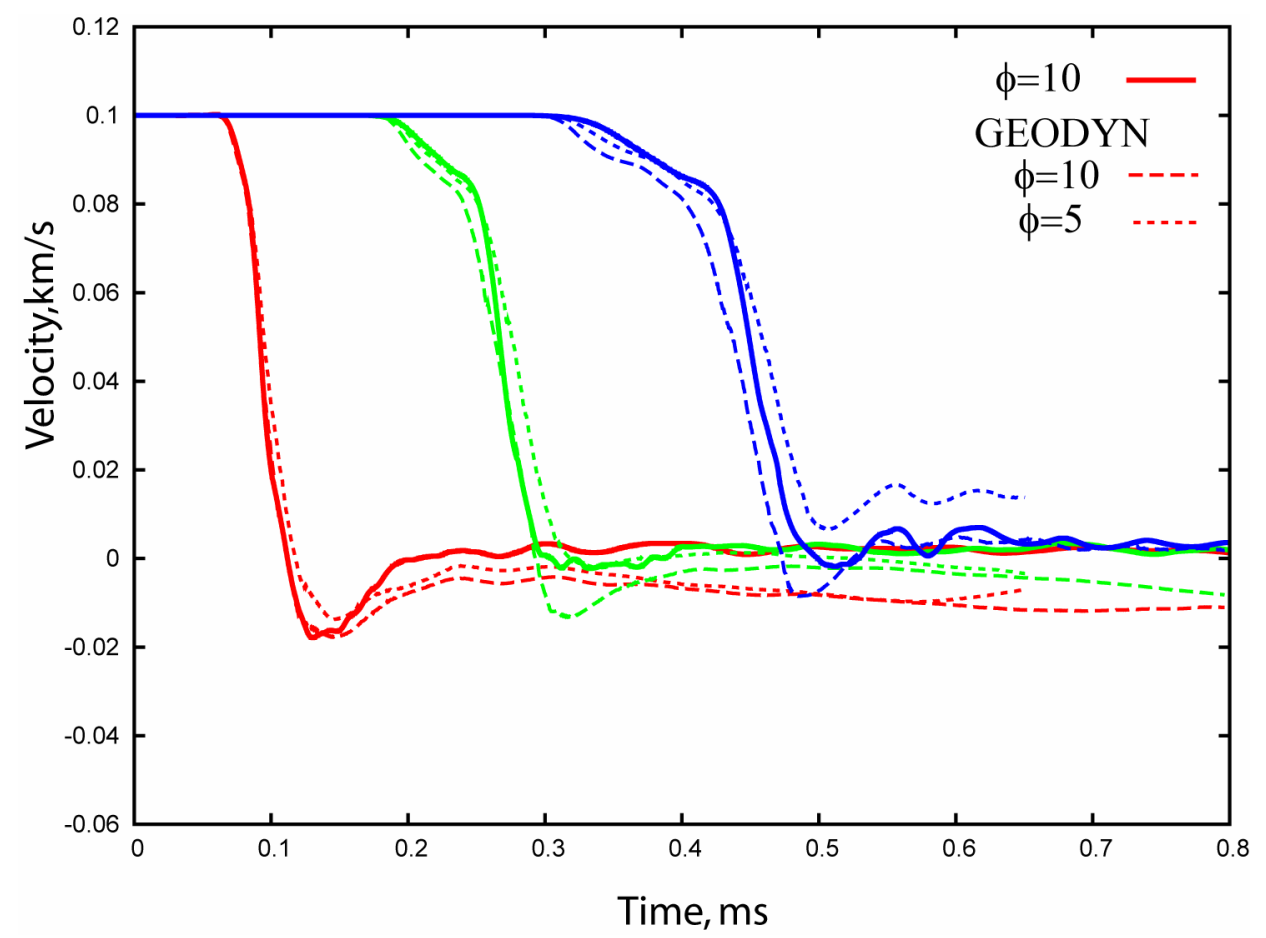

Figure.11 Calculated vertical velocity histories for points $0,1,2$. The friction angle is 10. The solid lines are Geodyn-L calculations, the dashed lines are GEODYN calculations with two different frictional angle values (5 and 10) 


\section{Conclusions}

The simple algorithm implemented in GEODYN for joint treatment is in good agreement with more accurate Lagrangian treatment available in Geodyn-L code, provided more than 4 cells span the joint.

To improve joint treatment further the following can be done:

1) If joint normal does not change orientation during the deformation or this change is insignificant an additional vector specifying this orientation can be added to the joint description, otherwise additional history variable for joints orientation will be required.

2) Using the joint orientation it should be possible to modify currently used additional plastic update making it directional (Note, that the currently isotropic update is used based on the cell pressure, rather than the normal stress). For that purpose, the strain and stress deviators can be reduced to satisfy conditions on the joint.

When it comes to very stiff noncompliant joints, the Eulerian treatment of joints implemented in GEODYN offers clear advantage in comparison to the explicit lagrangian treatment, because in the later case the time step is strongly limited by the joint stiffness.

\section{References}

[1] Lomov, I. and M.B. Rubin, Numerical simulation of damage using an elasticviscoplastic model with directional tensile failure. Journal De Physique Iv, 2003. 110: p. 281-286.

[2] Lomov, I., et al. Patch-based Adaptive Mesh Refinement for Multimaterial Hydrodynamics. in Five-Laboratory Conference on Computational Mathematics. 2005. Vienna.

[3] Vorobiev, O.Yu., 2007. Simple Common Plane contact algorithm for explicit FE/FD methods, LLNL report, UCRL-TR-227085. 